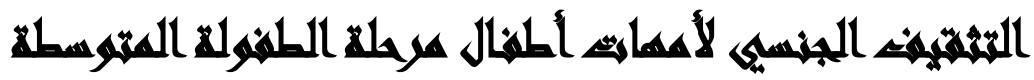

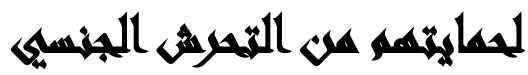

\section{[IY]}

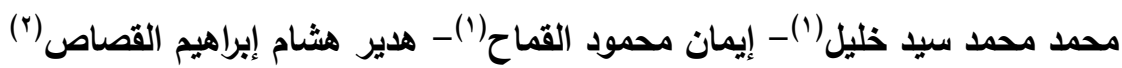

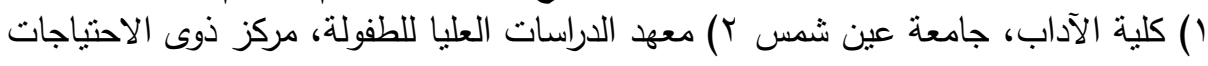

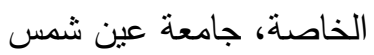

\section{المستخله}

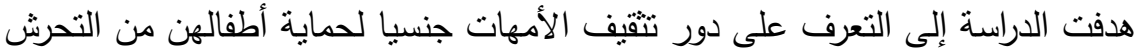
الجنسي، وأوضحت الدراسة أهمية اختيار مرحلة الطفولة المتوسطة ودور الأسرة في التنقيف

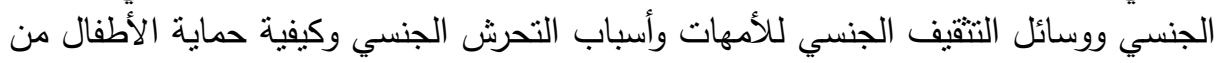

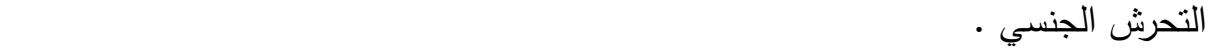
واستخلصت الباحثة من خلال عرض الدراسات السابقة والإطار النظري ان عدم توعية

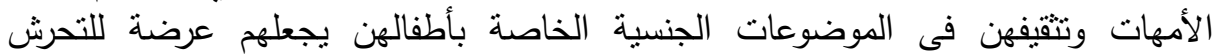

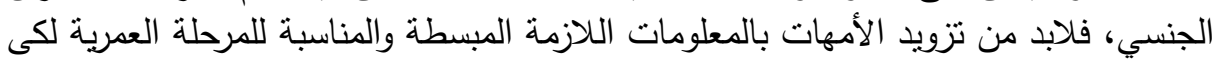

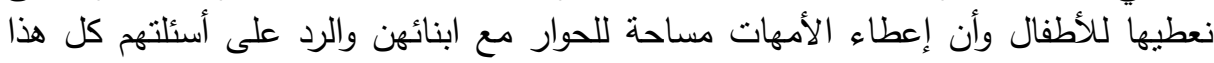
يقلل من خطر تعرضهم للتحرش الجنسي.

\section{zanadl}

يهتم المجتمع بالطفل وبنموه السوي ليصبح عند رشده إنساناً سوياً:حيث أن سنوات أندات الطفولة الأولى لها أهية في تتشئة الطفل وفي حياته المستقبلية حيث أن خبرات الطفولة تتنقل مع الفرد إلى المراحل اللاحقة ونترك بصماتها قوية راسخة في شخصية الراشد الكبير. فإذا كان النتشئة والنمو يسيران ويمران بشكل طبيعي إلى حد ما فيظهر ذللك في شخصية الفرد فيما بعد وإذا كانت تتشئته ونمو الطفل يوجد فيه بعض الاضطرابات فئن فإن ذلك يظهر أيضاً في

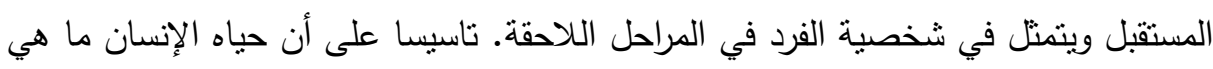

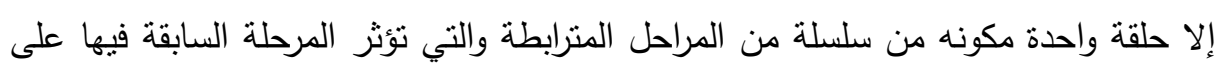

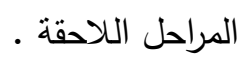

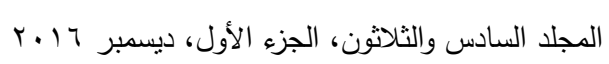


وقد أوضح صالح عبد الكريم أن الطفل في مرحلة الطفولة المتوسطة ينتقل في هذه المرحلة من الاعتمادية إلى الاستقلالية كما يتسم الطفل في هذه المرحلة باتساع الآفاق العقلية المعرفية حيث أن هذه المرحلة تتميز بما بطرأ على الطفل من تغيرات في جميع أنواع النمو لئه

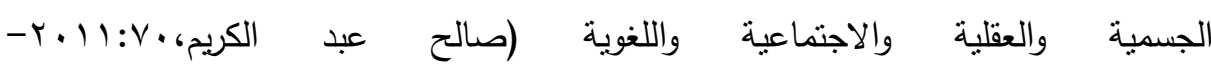
.(www.tge.gov.sa) $)(\vee q$

كما اشار Flanagan إلي أن من نتائج البحوث عن نمو الطفل نجد أفكارا عن براءة

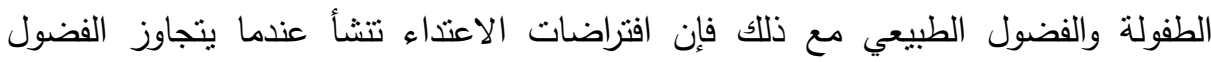

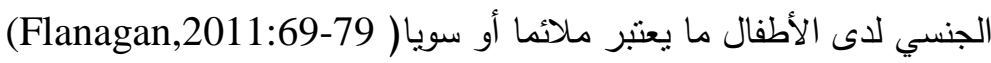

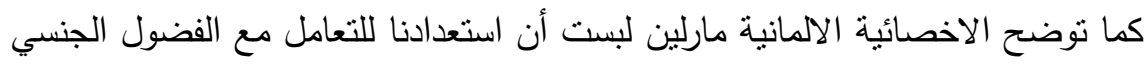

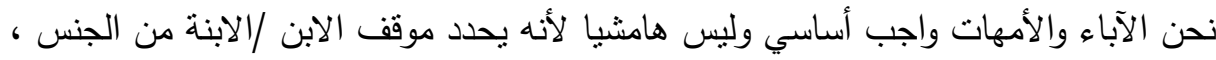

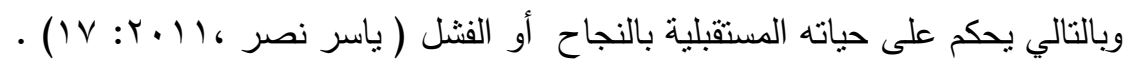

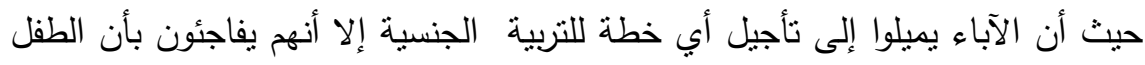

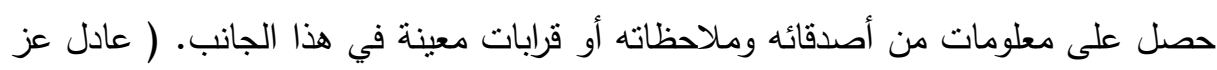

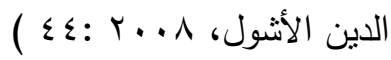
كما أن الحديث عن الحياة الجنسية في مجتمعنا من المحظورات، وأحيانا من الكبائر فهو مرتبط دائما بالعيب والحرام، وعلى الرغم من أنه يوجد أبعاد أخرى لهذا، فالحياة الجنسية ليست هي فقط العلاقة الحميمة بين الرجل والمرأة، بل هي تشمل كل ما يتعلق بالأعضاء التتاسلية للى الإنسان وما يرتبط بها من مشاكل وفترات نموه والنضج، وأيضاً أكد حتى وقت قريب كان موضوع النشاط الجنسي في الطفولة من المحرمات ولا يمكن التصريح

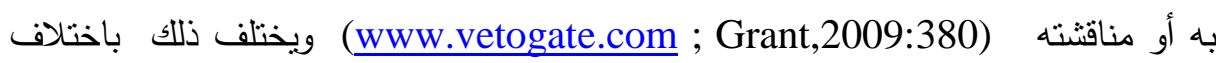
المجتمعات الحضارية والريفية والصحراء بمصر . بأه ولعل عدم وعي الأهل بالتحدث مبكرا مع أطفالهم حول هذه الموضوعات قبه فيل ذهابهم إلى الددرسة يجعلهم عرضه للإساءة الجنسية أو التحر الجنسي وبالإلنالي ينصح الأهل

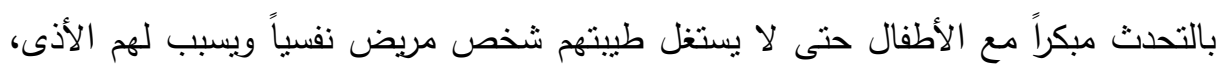


كما من المهم أن يكون الأهل المرجع الوحيد للطفل حول هذه الأمور، ويجب أن يبني الأهل ثقة منبادلة مع الطفل حتى يستطيعوا السيطرة على جميع اسئلته فيما بعد (هاله ابراهيم

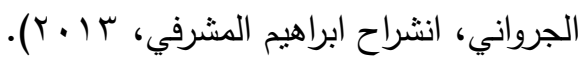
حيث أثارت نتائج دراسة Branham 2012 إلى أن الآباء والأمهات بحاجة إلى الى

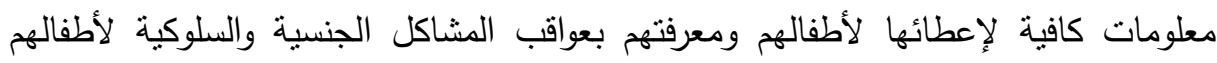

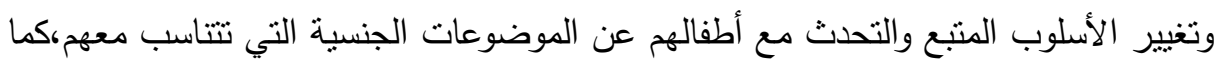

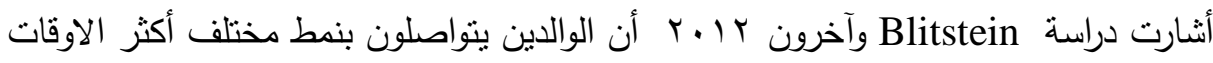
في الموضوعات الجنسية.

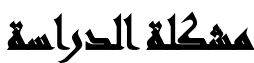

أوضحت الدراسات الإحصائية مدى انتشار ظاهرة التحرش والاعتداء الجنسي والمشكلات الجنسية ، " ففي مصر جاءت حوادث الاعتداء الجنسي على الأطفال في المرنبة

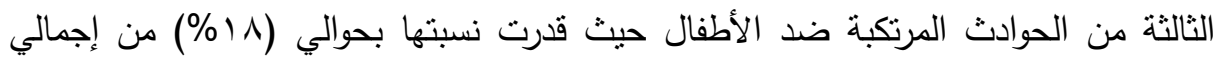
الحوادث المختلفة للأطفال وفيما يتعلق بصلة مرتكب حادثة الإساءة الجنسية للأطفال فقد أتضح أن نسبة هr\% لهم صلة قرابة بالأطفال، ونسبة (؟\%) ليس لهم صلة بهم" • (منيرة

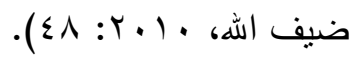
أما بالنسبة لعدم وجود الوعي الجنسي فلم تجد الباحثة إحصائيات في حدود علمها. ولكن

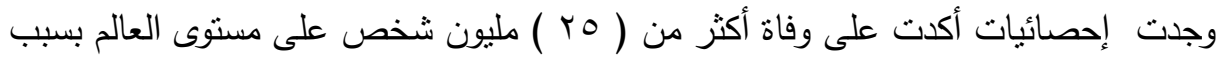

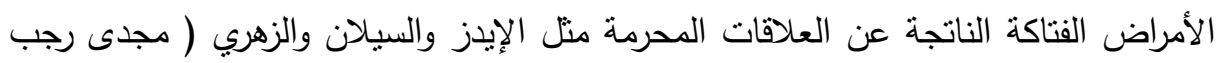
$\cdot\left(\Lambda \Lambda: r_{\cdots} \cdot v_{6}\right.$ ولعل تحفظ الآباء عن الخوض في التحدث عن الثقافة الجنسية مع أبنائهم نابع من نوع التهاء

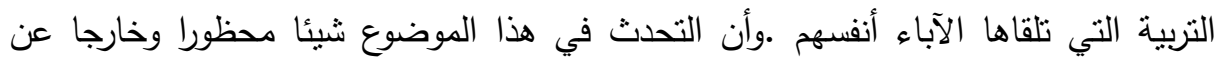

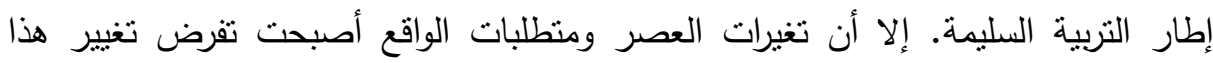

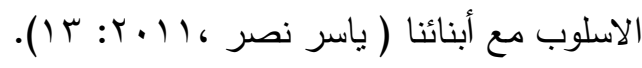


حيث أوضح نتائج دراسة Walsh وآخرون ( r ب ـ ) عن أن التواصل بين الأم والطفل

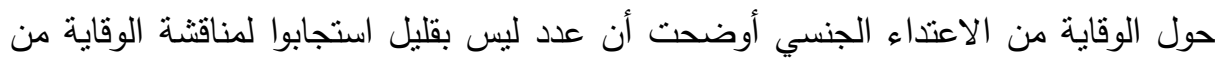

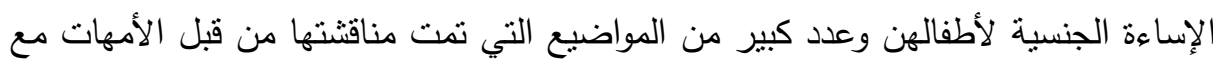
أطفالهم. في حين تتاولت دراسة woody وآخرون (0. . . ) جهود الأمهات نحو تعليم الأطفال

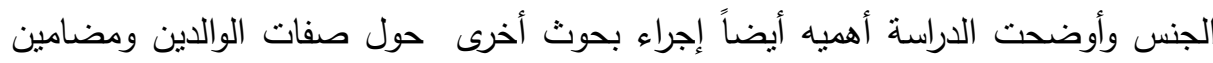
لتصميم برامج لمساعدة الوالدين في لعب دور أكبر في تربية أطفالهم الجنسية.

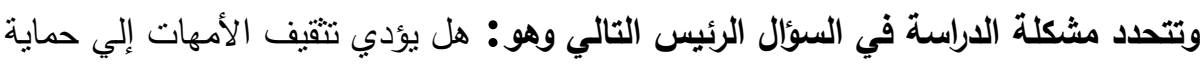

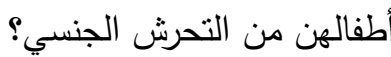

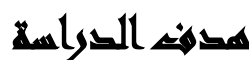

يتحدد هدف الدراسة في رفع المستوي الثقافي للأمهات فى مجال حماية أطفالهن من التحرش الجنسي.

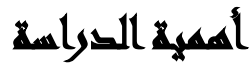

تركز الدراسة على مرحلة الطفولة المتوسطة والتي تتم فيها عمليه التنشئة الاجتماعية

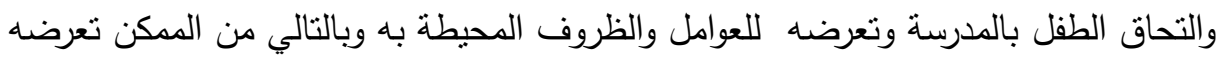

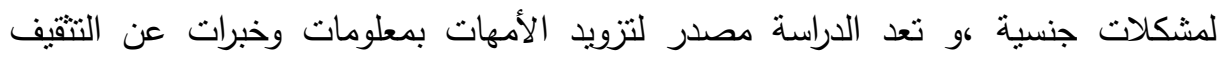

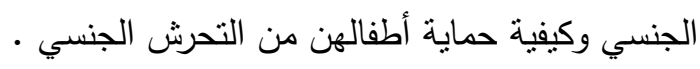
ويتحقق هدف الدراسة من خلال عرض مفاهيم الدراسة ومن خلال عرض عن الإن الإنار

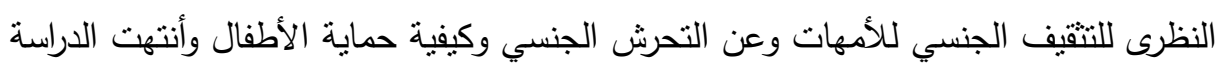

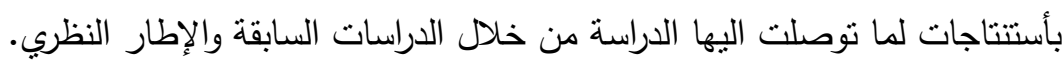




\section{مخاهيه الصراسلة الأساسية}

1-التثقيف الجنسي للأمهات: هو الإطار القيمي والأخلاقي المحيط بموضوع الجنس

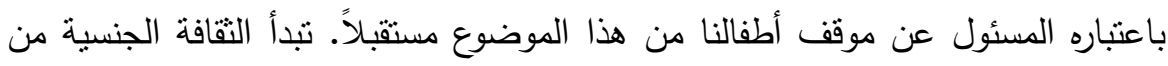
تعرف الطفل على جسده وفهم خريطته ومراحل النمو الجسمي وتغير الأعضاء والفرق في ذلك بين الولد والبنت أو الرجل والمرأة فيما بعد وإعطاء معلومات مبسطة وفرلة وحقيقية عن

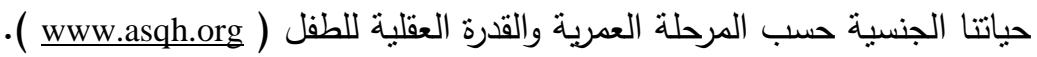
r-مرحلة الطقولة المتوبطة: وهى المرحلة التي يلتحق فيها الطفل بالمدرسة الابتدائية إما قادما من المنزل مباشرة أو منتقلا من رياض الأطفال' وهي تشمل الصفوف الثلاثة

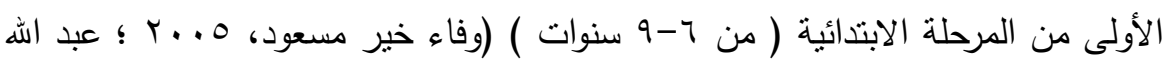

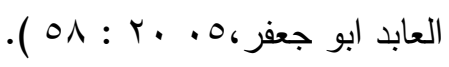

r- التحرش الجنسي: هو مضايقة، أو فعل غير مرحب به من النوع الجنسي، يتضمن

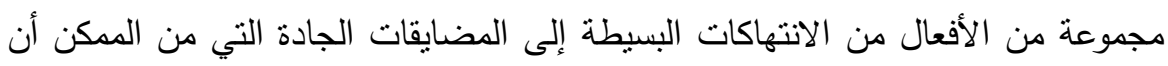

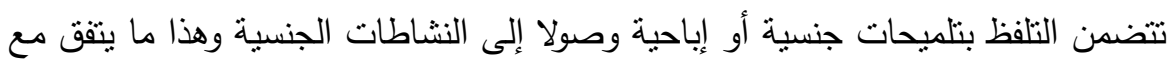

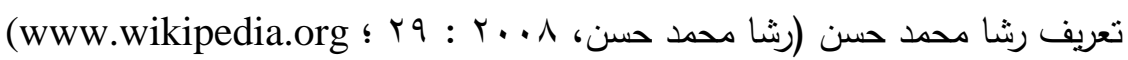

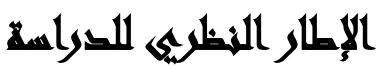

مرحلة الطقولة المتوسطة: وتم اختيار هذه المرحلة حيث انها تحدث فيها عملية التنشئة الاجتماعية والتحاق الطفل بالمدرسة وتعرضه للعوامل والظروف المحيطة به وبالتالي من

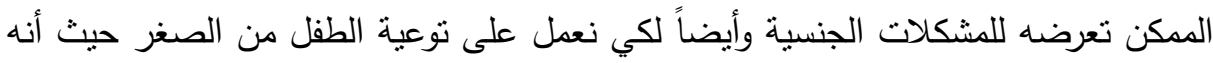

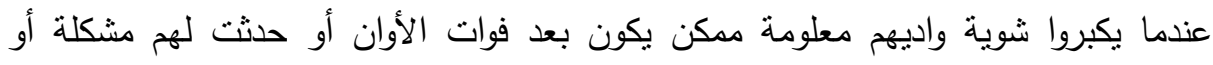
تعرضوا لشيء لكن إذا لحقنا من الأول سيقلل من احتمال وقوع المشكلات. 
وأيضاً في مرحلة الطفولة المتوسطة يزيد حب الطفل للاستطلاع والتعرف والقدرة على التذكر بشكل أفضل حيث أنه يدرك كثير من الأثنياء ومنها إدراك الأثكال وعلاقتها المكانية

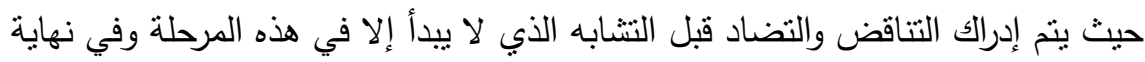

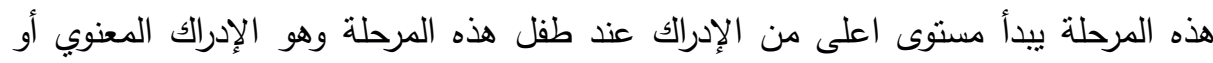
المجرد إذ يميز بين الصواب والخطأ والثر والخير وتزداد علاقاته وروابطه الاجتماعية ويتحدد الإدها

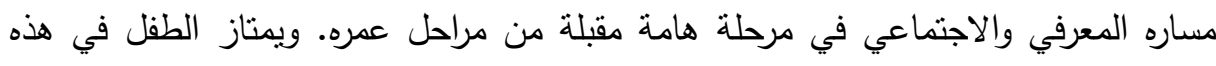
المرحلة بقدرته المتفوقة على التذكر ويعتمد الطفل في هذه المرحلة على الذاكرة التي تتميز بأنها الية ومباشرة كما أنه بمكنه تذكر الأثباء المجردة.

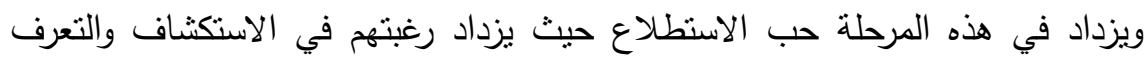

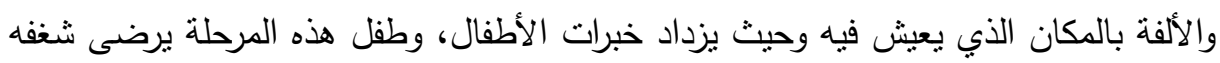

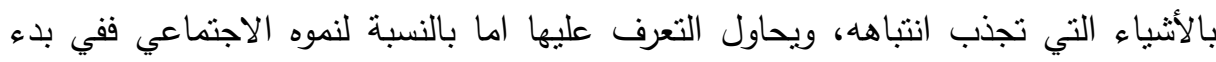

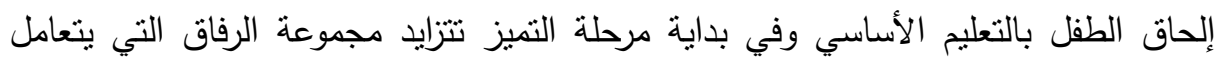

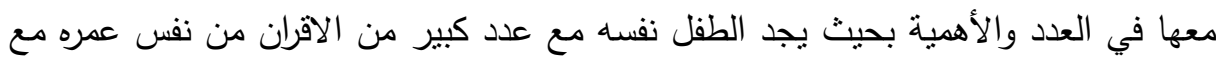

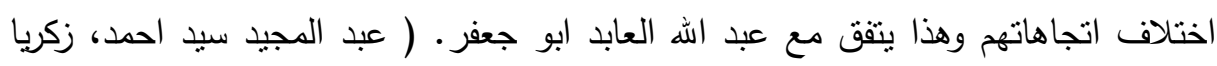

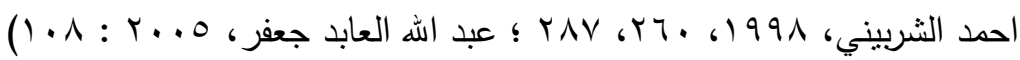
التثقيف الجنسي للأمهات: ولابد أن نذكر أن الثقافة الجنسية ضرورية للأسرة وللآباء والابناء،

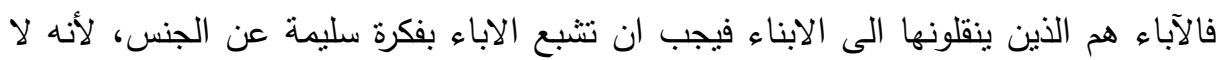
فائدة من إدخال التربية الجنسية في المدارس اذا ما كان البيت يعارضها، ولكى تتحقق التربية

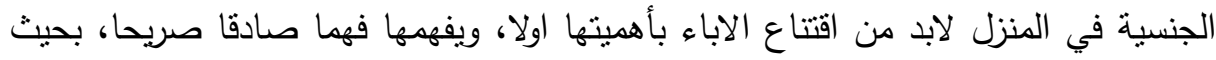

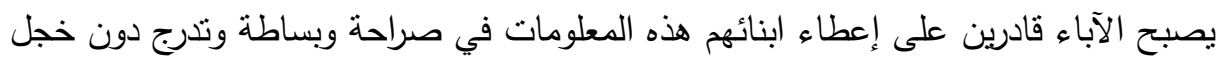

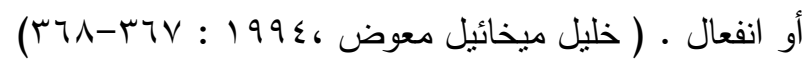

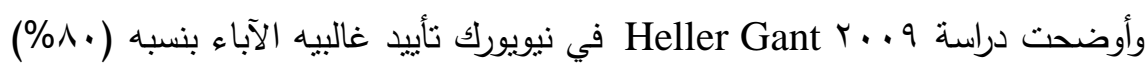
من (191 ) من الآباء في احدى كليات المجتمع الحضري تأييد موضوعات التربية الجنسية كانت هذه النتيجة مفاجأة لان العينة كانت منتوعه في ضوء الاء العرقية فقد هاجر غالبيتهم من 
دول تعتبر محافظه اجتماعيا واتفقت النتائج مع نتائج الاستطلاعات المستبقة على مستوى الولاية والمستوى الوطني.

ويرى ياسر نصر اننا من المكن أن ننمى الثقافة الجنسية لدى الطفل دون ان نخش ولش

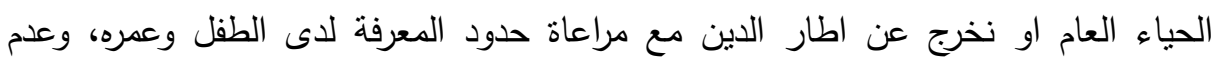
الاستخفاف بدرجة فهمه .وذللك عن طريق: الاجابة على اسئلة الاطفال بأكبر درجة من الصدق ودون الاخول في التفاصيل مع مراعاة

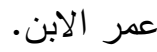
تشجيعهم على السؤال وعدم تخويفهم من شبح العيب وكذللك عدم اظهار الحرج والقلق اثثاء الاجابة على هذه الاسئلة. في حالة المرور بموقف جنسي امام الطفل والوالدان موجودان فيجب على الوالدين الثرح المبسط والمختصر لما يحدث وعدم نرك هذا الموقف لمخيلة الطفل. وضع قيود على نوعية الاعلام الذى يتلقاه الطفل، مثل بعض الافلام التي تعرض برض مشاهد جنسية عدم اهمال مرور الطفل بحالة نفسية سيئة، فقد يكون الطفل قد تعرض لتحرش جنسي

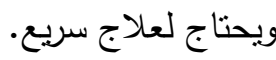
يجب مراعاة حجم الجرعات من المعلومات التي تعطى للطفل ومراعاة السن.( ياسر نصر، (1) ب. (10-7) حيث توضح أميرة جمال أن إجابتتا على الطفل تمثل مسئولية كبيرة قد تقيده او تضره ، فيمكن ان تكون اجابتتا خاطئة فنعرضه للخطر ، ولا نعتبر أن الطفل عندما يسألنا انه كثير السؤال وثرثار وانما هو طفل ذكى • ولابد ان ننظر إلى الطفل باحترام عند سؤاله ونسأل انفسنا

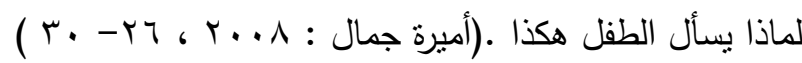

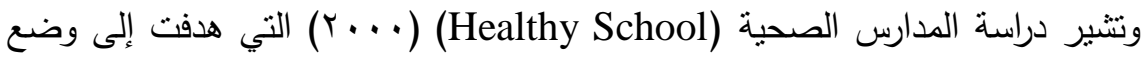

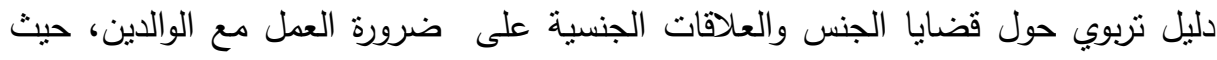
تبين أن الوالدين يعلمان أطفالهم أمور الجنس والعلاقات الجنسية، كما أنهح يساعدان أطفالهم

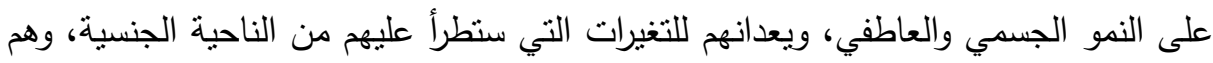


الأقدر على الإجابة عن أسئلة أطفالهم الجنسية، وأوصت الدراسة بضرورة دعم الوالدين حتى يقوموا بهذه المهمات على أكمل وجه.

وأيضاً يتضمن دور الاسرة في أشياء يجب على الوالدين تعلمها لأولادهم في مرحلة الطفولة

المتوسطة:

1- تعليمهم أن مواطن العورة لابد من سترها، ولا يجوز إظهارها ابدا إلا للحاجة الملحة من قضاء الحاجة، أو عند الاستحمام.

r- لزوم وجوب الاستتجاء والتطهر من البول وإرشادهم إلى حرمة النظر إلى عورات بعضهم البعض، ومتابعتهم في تحقيق ذلك.

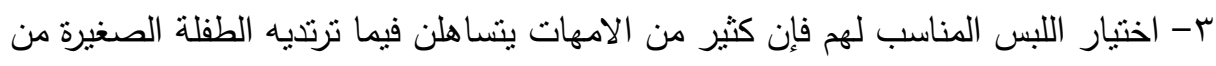
ثثاب قصير قد تظهر منها عورتها فتكون عرضة للاستغلال من قبل الاخرين، وكذللك

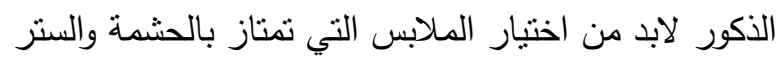

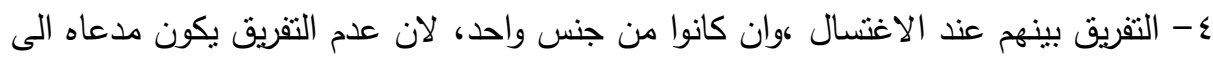
النظر الى العورات، واعتياد ذلك بين الاطفال يسقط هيبة غض البصر في نفوسهر هن

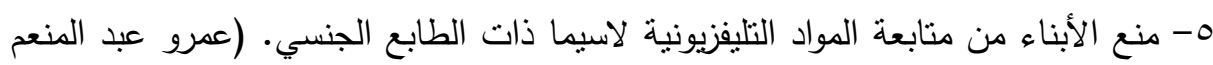
بالتالي نحتاج التربية الجنسية أهميتها في أنها تجعل للأطفال: 1- تعرفهم على جانب هام من جوانب نموهم المختلفة

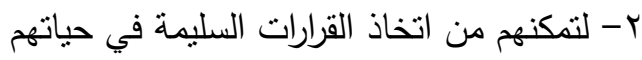
ب- لامتلاك القدرة على التكيف مع ضغنط الزماد التهاء والأخرين

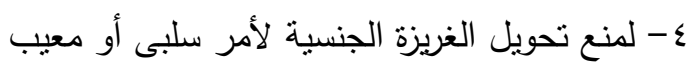
0- لحمايته من الاستغلال الجنسي وغيره 4- لـنعهم من الاعتداء على الأخرين

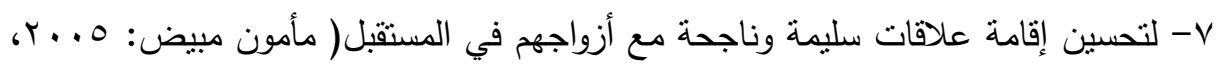


كما رأت دراسة Corngold ^ . . ب أنه في المجتمع الحر يجب أن نتاح للآباء فرصه عرض وجهات نظرهم حول الجنس والأخلاق الجنسية لأطفالهم مع ذلك فإن الاهتمامات التي لته

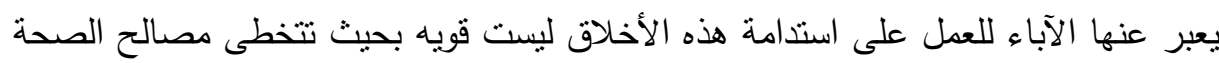

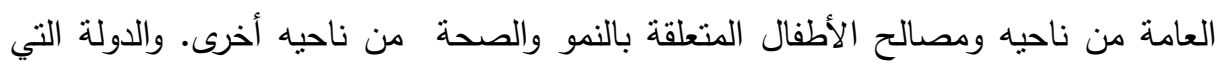
تفرض على كل المدارس تزويد طلابها بتربية جنسية شاملة نتشمل المعلومات الطبية الدقيقة

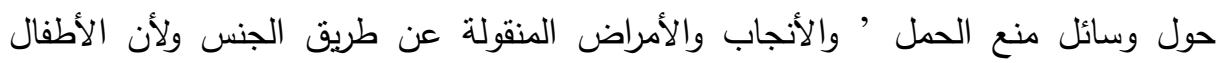
والمراهقين لديهم اهتمام أساسي أن يصبحوا مستقلين ذاتيا، وراشدين يحترمون أنفسهم، ولأن النشاط الجنسي هو مجال فيه يكونوا سهل التعرض للتناعب والاستغلال والاعتداء ، فإن تعليم الجنس الذى تفرضه الدولة يجب أن يتجاوز مجرد تزويد المعلومات لعلاج الأبعاد الوجدانية

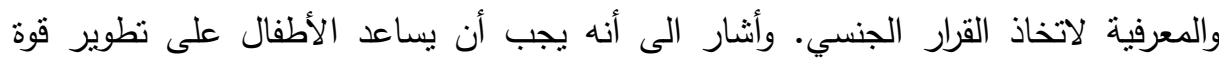

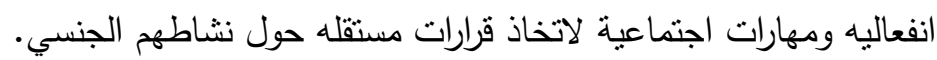
أسباب التحرش الجنسي وتتمثل فيما يلي:

1. نقص التوعية الجنسي المطلوب نوفيرها للأطفال في مختلف الأعمار .

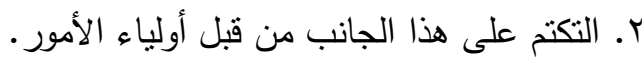
r. حب الاستطلاع الذي يتميز به بعض الأطفال مما يجعلهم فريسة سهلة.

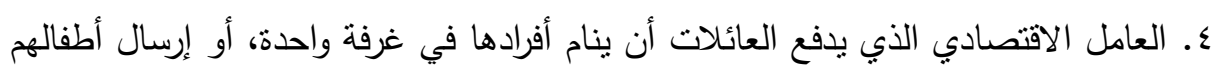
للعمل في أماكن غير آمنة. ه. عدم مراقبة الوالدين للأطفال لما يشاهده عبر أمبر وسائل الإعلام.

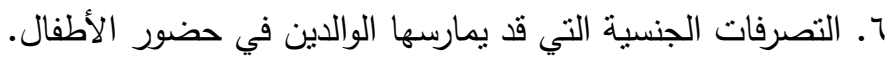

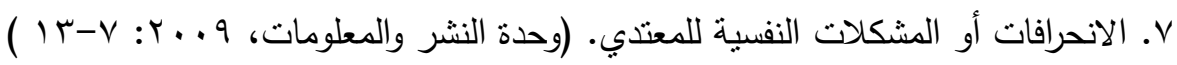


كما أوضحت نتائج دراسة جنان أ. أسطا واخرون A ... الى رجوع النساء أسباب الاساءة الى الافلام الاباحية والصور المثيرة وتفكك الأسرة ( العنف المنزلي او عدم مراقبه

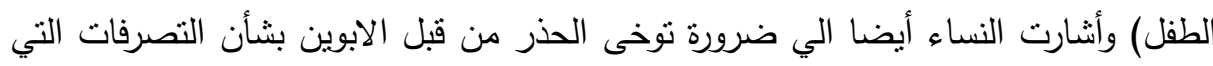

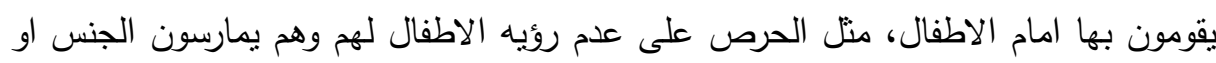

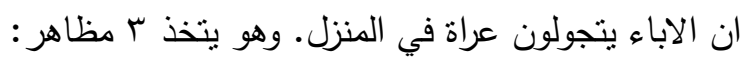
1-تحرش جنسي شفوي ( لفظي ) ( ملاحظات وتعليقات غير مثنتة، طرح اسئلة جنسية ) r-تحرش جنسي غير شفوي ( غير لفظي ) مثل نظرات ايمائية

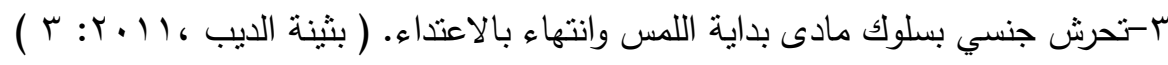

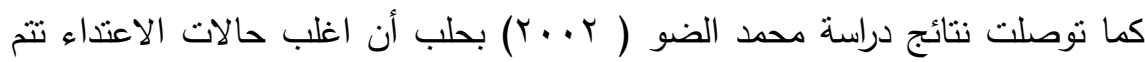
من قبل أثخاص يفترض أن يؤمنوا لحمايه للطفل وفي أماكن يفترض أن تكون المكان الامن للطفل وان غياب التوعية والثقافة الجنسية في المجتمع يجعل الكثير من الاطفال يتعرضواليها للاعتداء دون أن يعرفوا أنهم تعرضوا للاعتداء كما أوصت بإيجاد الوعى الأسرى بالتربية الجنسية ومساعدة المؤسسات التعليمية الاجتماعية وتبنيها لهذا الجانب منذ سنوات الطفل الاولي على أن تكون الأسس مستمدة من الاسلام.

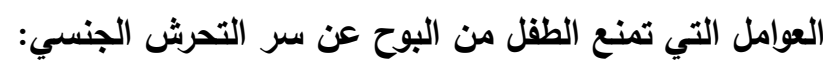

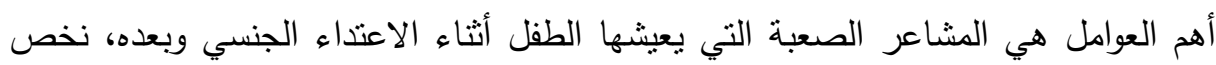

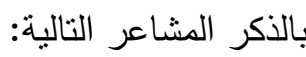

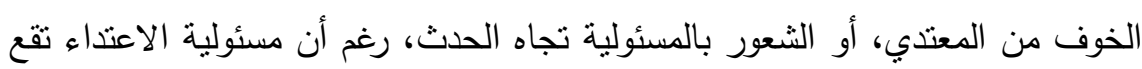

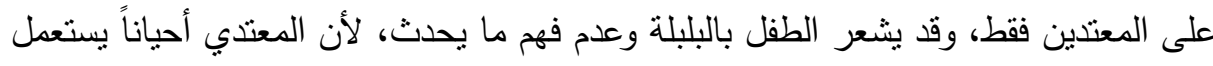

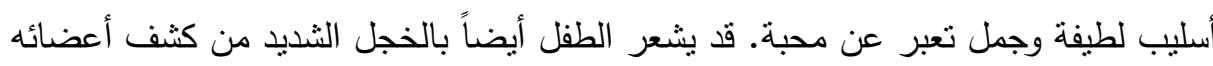

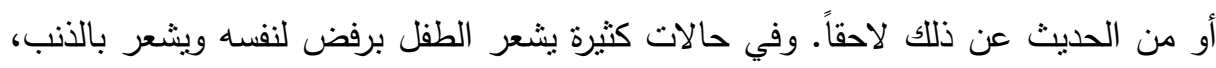

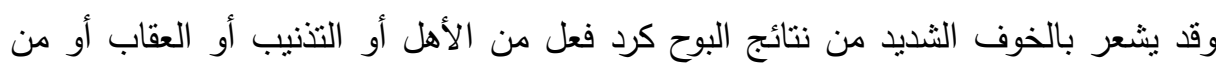

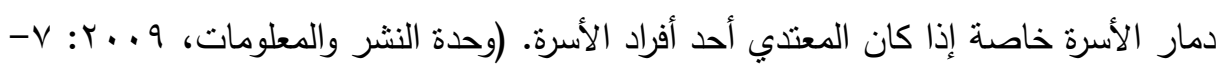




\section{كيف يحمي الطقل نفسه من الاعتداء والتحرش الجنسي:}

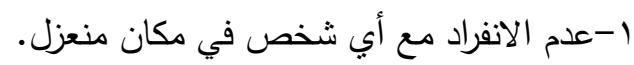

r-ترك مسافة متر واحد تقريباً بين الطفل وبين المتحدث أو الثخص فئن المعتدي r- زعزعة ثقة الثخص بنفسه وتخويفه.

ع - تبليخ الوالدين بما حدث لأنهما سوف يدافعة وتهان عن الطفل.

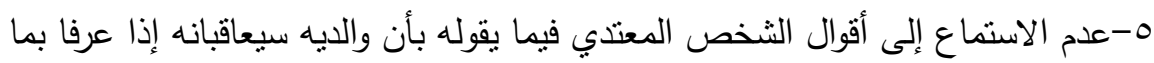

$$
\text { حدث. }
$$

1- زرع الثقة والثجاعة في نفس الطفل لأنه هو الأقوى

$$
\text { V - عدم الانجراف وراء الإغراءات المادية والمعنوية. }
$$

^- غرس روح الدفاع في نفس الطفل وأن الثخص المعتدي جبان لا يقدر على فعل شيء.

$$
\text { 9-إظهار حب الوالدين لطفلهما بصورة مستمرة. }
$$

. 1 - تعليم الطفل وتفهيمه أن جسمه ملكُ" خاص له. له.

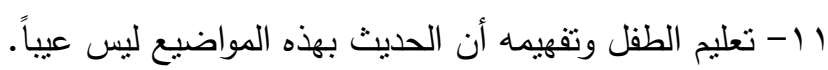

r r-عدم تلبية الطفل لنداء أي شخص يسأله أن يرافقه.

ب ا --عدم الذهاب إلى الأماكن العامة بمفرده.

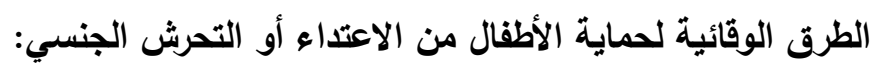

1-إذا حاول الثخص المعتدي أن يمسك الطفل من الخلف ويده على فم الطفل:

$$
\text { أ- استغلال مواضع القوة عند الطفل. }
$$

$$
\text { ب- استغلال مواضع الضعف عند الثخص المعتدي. }
$$

r-الصراخ: الصراخ بنبرة صوت حادة من البطن مع استخدام كلمات وهمية تخويفية.

r- نرك مسافة بين الطفل والثخص المعتدي الذي أمامه. وتضيف national sexual violence إلى أنه لابد من تزويد الطفل بمعلومات عن من لطن التطور الجنسي ومفهوم المناطق الخاصة، وأي المناطق (الأعضاء) في الجسم يمكن اعنبارها

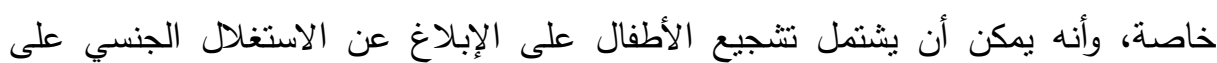

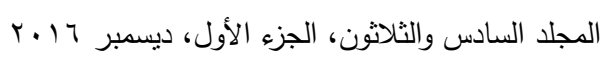


*الإستغلال الجنسي للأطفال ليس أبدًا خطئهم. *إذا قام أحد الأشخاص بلمسهم بشكل غير مريح عليهم أن يخبروا دائماً شخصاً موثوثًا فيه. ع - لا ينبغي ألا يحفظوا سراً عن قيام أحد أبّا كان بلمسهر.

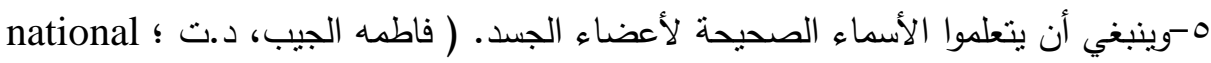

( sexual violence ,2011: 2-6

\section{اللعامي}

من خلال عرض الدراسات السابقة والإطار النظري يمكن استخلاص من كل ما سبق عرضه أن النتقيف الجنسي للأمهات وتوعيتهن تقلل من خطر التحرش الجنسي للأطفال الذين

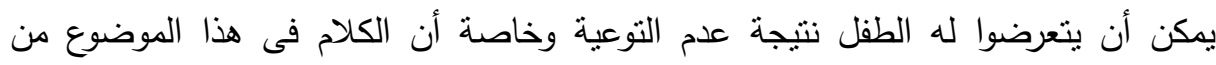
المحظورات ونتجنب التحدث في منل هذه الموضوعات، وايضا رد الأمهات على أسئلة

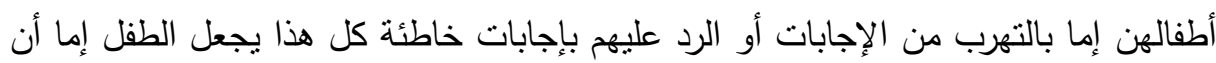

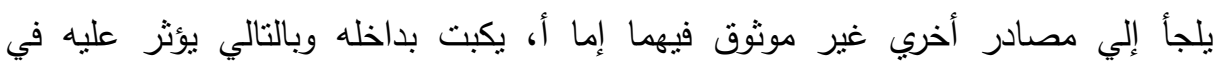
المستقبل. - الم

حيث أوضحت الدراسات السابقة أن الأمهات بحاجة إلي معلومات كافية لإعطائها

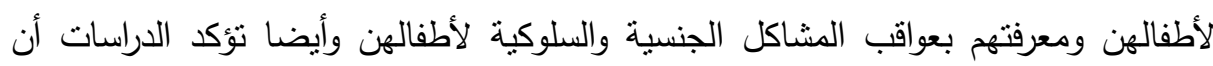
الأمهات يتواصلن بنمط مختلف فى الموضوعات الجنسية عن باقي الموضوعات، وأن معظم

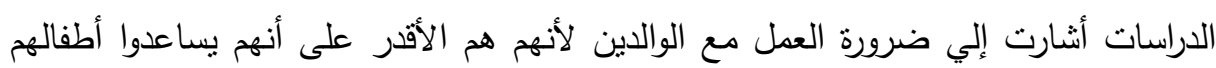

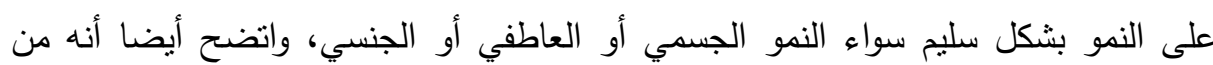

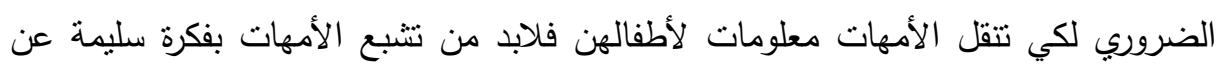

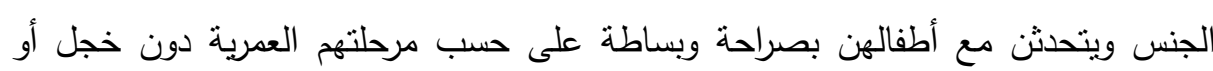

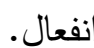

واتضح ايضا أنه اذا تدربت الأمهات واشتركن في حلقات البحث، الندوات عامة أو

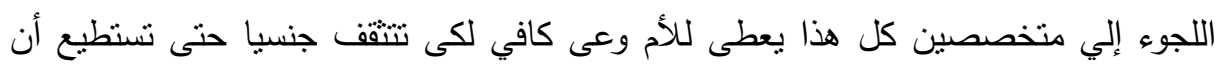

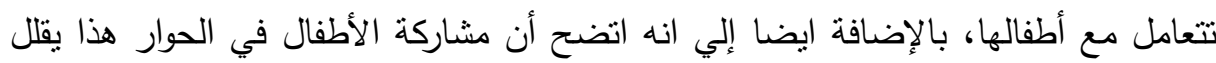
290

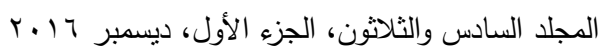


ايضا من حدوث التحرش الجنسي حتى إن حدث له شيء لا يخجل ويحكى للأم فتبدأ في التصرف أفضل من أنه لا يخبر الأم أي شىء لأنها لنها لن تسمح له بالتحدث في هذه الموضوعات.

واذا نم بتفعيل دور الأسرة في النقيف الجنسي فنعلم الطفل الخصوصية عند تغير ملابسه أمام الاخرين أو الأقارب والاعتماد على النفس ومتابعته في مشاهدة المواد التلايفزيونية. وتعليمه انه غالي وكيفية حماية نفسه ومتى يقول لا كل هذا يقلل من تعرضه لخطر التحرش الته

وأيضا حينما تدرب الأمهات على ان يدربن أطفالهن على احترام أجسادهم وخصوصيته

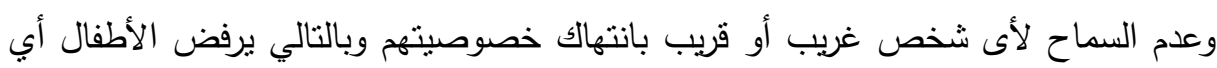

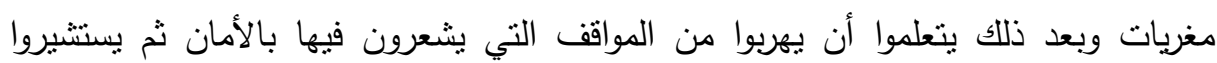
بمعنى ندريبهم اذا حدث لهم انتهاك جنسي فلابد من ابلاغ الأسرة.

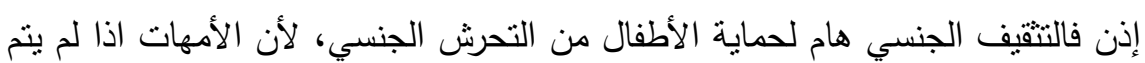

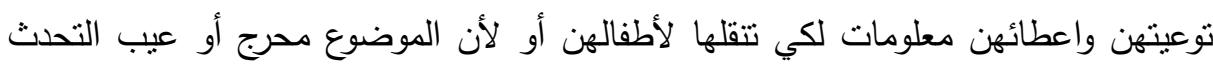
في ' فمن المكن أن تحدث المشكلة وبعد ذلك تحدث مشكلات نتيجة عدم نوعية الأطفال

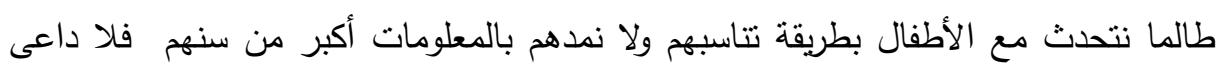
• للإحراج

حيث أكدت الدراسات أن معظم الأمهات وافقن على تدريس التربية الجنسية نظرا لما

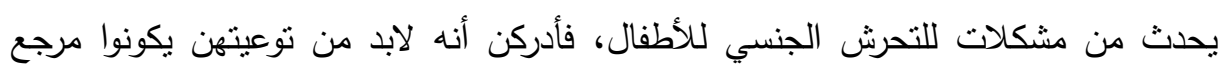
لأطفالهم وتدريبهم على حماية أنفسهم.

\section{المرأar}

أميرة جمال(^ . . ب): أسئلة طفلك الحرجة وكيف نجيب عنه؟، القاهرة، مؤسسة اقرأ

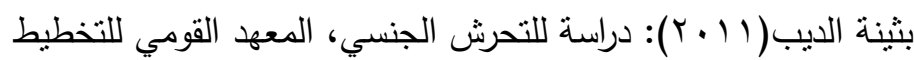

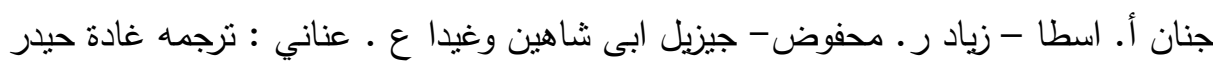

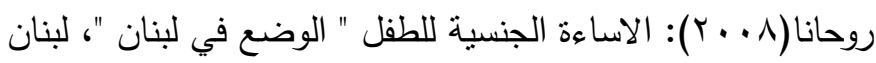

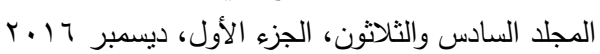


خليل ميخائيل معوض(ء99 (1): سيكولوجية النمو (الطفولة والمراهقة)،طس، دار الفكر

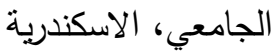

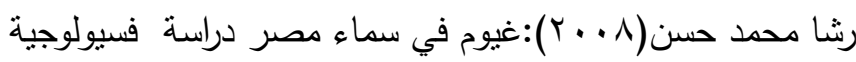

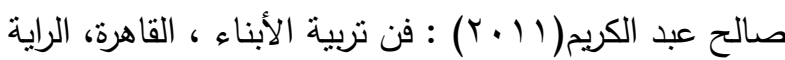

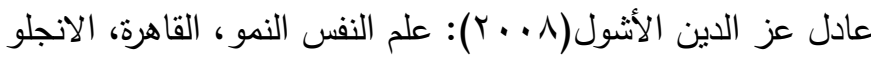
عبد الله العابد أبو جعفر(0. ․ ץ): علم النفس النمو، الوثيقة الخضراء، ليبيا عبد المجيد سيد أحمد، زكريا احمد الثربيني(991)(1): علم النفس الطفولة الاسس النفسية

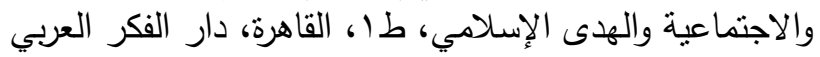

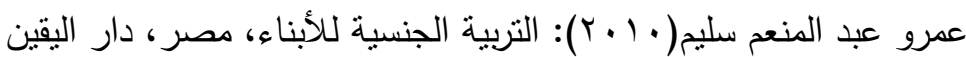
فاطمة الجيب،(ضابطة تمريض للمنطقة الثانية)، نصائح ذهبية للآباء والأمهات لتدريب

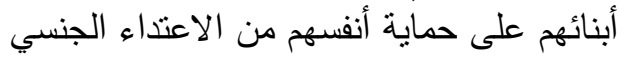

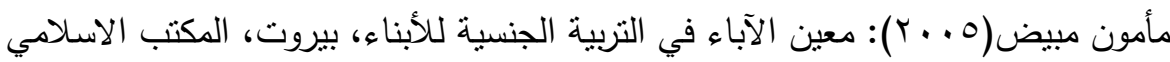

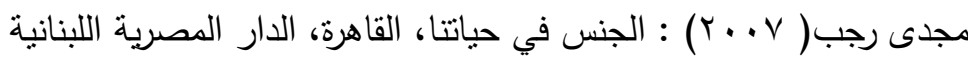

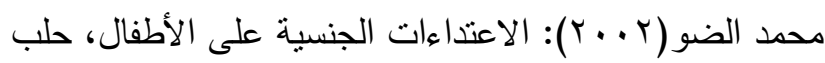

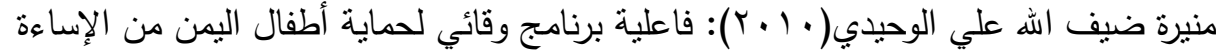
الجنسية، رسالة ماجيستير ،معهذ الدراسات العليا للطفولة، جامعهد عين شمس.

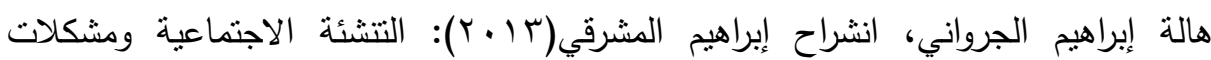

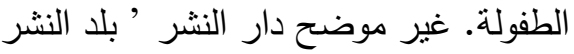

وحدة النشر والمعلومات(9 + . ץ): دراسة حول وقع الاعتداء الجنسي ،غزة .

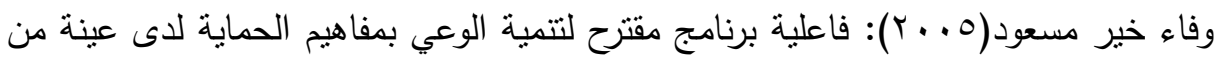

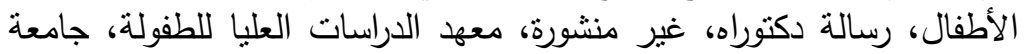

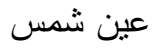

ياسر نصر (1) (1): تربية الأبناء على الثقافة الجنسية، القاهرة ، مؤسسة بداية 
Blitsein,G.1 ; Evans , W.D ; Davis ,K.C \& Kamyab.K.(2012) : Rpeated Exposure to media messages encouraging parent - child communication about sex , journal of health promotion, 27 (1): $43-51$.

Branham ,C.R .(2012) : Birth mother's Perceptions of what parents need to know to educate their pre - adolescent child about human sexuality. Humanities and social science,73(6A):23-40.

Corngold, J.(2008). Toleration, parents' rights, and children's autonomy: The case of sex education.Stanford University, ProQuest, UMI Dissertations Publishing,. 3332812.

Flanagan,p.(2011). Making Sense of Children's sexualityunderstanding sexual development and activity in education contexts.Journal of education, 16(3):69-79.

Grant,R.k;Lesley,h;and Kingston,n.(2009).intervention for children with sexual behavior problem. Civic research in statute, 380 .

Health school.(2000). Sex and relationship education guidance, producted by the department for education and employment, England

Heller, J.(2009) .The relationship between parental opinion of schoolbased sex education, parent-child communication about sexuality, and parenting styles in a diverse urban community college population.City University of New York, ProQuest, UMI Dissertations . 3378576.

National Sexual Violence Resource Center (2011): Child Sexual Abuse Prevention Programs for Children.

Walsh,K; and Barandon ,L.(2012): Mother-child communication about sexual abuse prevention, 21(4) :399-421.

Woody,j.d;randal,a.d;and d"souza,h.j.(2005). Mothers' efforts toward their children's sex education. journal of family studies, 11(1):83-97.

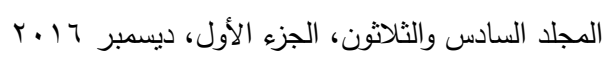


http://www.wikipedia.org

www.asgh.org

www.tge.gov.sa

http://www.vetogate.com / 26/6/2013-7pm

\title{
THE SEXUAL EDUCATION OF THE MOTHERS OF MIDDLE CHILDHOOD CHILDREN FOR PROTECTING THEM AGAINST HARASSMENT
}

\author{
Khalil, M. M. S. ${ }^{(1)}$; EIKammah, EMAN, M. ${ }^{(1)}$ \\ and Elkassas, Hadeer, $\mathrm{H}^{(2)}$
}

1) Faculty of Arts, Ain Shams University. 2) Institute of Postgraduate Childhood Studies, Center of Those with Special Needs, Ain Shams University.

\begin{abstract}
This study aims to identify the role of educating the mothers sexually for protecting their children against harassment. It highlights the importance of choosing the Middle childhood period, the role of family in sexual education, methods of mothers' education, reasons of harassment and how to preserve our children against sexual harassment.

Through reviewing the literature, and the theoretical framework, the researcher concluded that in case of not educating mothers sexually about their children, the children will be prone to sexual harassment. Accordingly, by providing mothers with needed simplified information suitable for their age group, and allowing a space of dialogue to mothers with their children to reply to their answers, will lessen the risk of exposing them to harassment.
\end{abstract}

$$
\text { المجلد السادس والثلاثون، الجزء الأول، ديسمبر r } 17
$$

